\title{
High Prevalence and Conservative Management of Acute Cholecystitis during Lenvatinib for Advanced Thyroid Cancer
}

\author{
Marta Di Stefano ${ }^{a}$ Carla Colombo ${ }^{a, b}$ Simone De Leo ${ }^{a}$ Michela Perrino $^{a}$ \\ Mauro Viganò ${ }^{c}$ Luca Persani ${ }^{a, d}$ Laura Fugazzola ${ }^{a, b}$ \\ aDivision of Endocrine and Metabolic Diseases, IRCCS Istituto Auxologico Italiano, Milan, Italy; ${ }^{b}$ Department of \\ Pathophysiology and Transplantation, University of Milan, Milan, Italy; 'Division of Hepatology, San Giuseppe \\ Hospital Multimedica IRCCS, Milan, Italy; ${ }^{d}$ Department of Clinical Sciences and Community Health, Milan, Italy
}

\section{Keywords}

Lenvatinib · Thyroid cancer · Cholecystitis · Weight loss

\begin{abstract}
Introduction: Lenvatinib (LEN) is a multitarget tyrosine kinase inhibitor currently used for advanced, radioiodine refractory differentiated thyroid cancer (RAI-R DTC). Among adverse events (AEs), nausea, vomiting, and decreased appetite have been frequently described. We aimed to evaluate the prevalence, the clinical presentation, and the effectiveness of conservative treatment of gallbladder disorders in a consecutive series of patient treated with LEN. Methods: Patients with RAI-R DTC experiencing clinical symptoms suggestive for gallbladder disorders during LEN treatment were evaluated with laboratory investigations and contrast-enhanced abdominal computed tomography (CT) and ultrasound scan (US). Results: After a median time of 2 months from the start of treatment, 5/13 patients (38.4\%) complained of gastrointestinal symptoms, with increased biliary enzymes levels, especially $\gamma \mathrm{GT}$, and CT/US suggestive of
\end{abstract}

acute cholecystitis (AC). The onset of symptoms and the peak of $\gamma \mathrm{GT}$ levels frequently corresponded to the highest reduction in body weight during the first months of treatment. All patients were treated with supportive care and, when appropriate, with ursodeoxycholic acid; in 4 patients, LEN dose reduction or short interruption was needed, too. Conclusions: In patients with RAI-R DTC treated with LEN, a high prevalence of $A C$ in the first months of treatment was documented. Mainly due to the low specificity of symptoms such as anorexia, nausea, and vomiting, this AE is likely to be frequently misdiagnosed. The onset of the disease was associated to the weight loss observed during the first months of treatment and contributes to further decrease in body weight. Therefore, particularly during the first months of treatment, or at any time of huge reduction of body weight, monitoring of $\gamma G T$ and US is crucial for prompt diagnosis and treatment. Conservative medical treatment and LEN dosage titration, together with dietary and rehabilitative supports, can limit or avoid the need for drug withdrawal and cholecystectomy.

(c) 2020 European Thyroid Association Published by S. Karger AG, Basel

\section{Karger $\stackrel{2}{\circ}$}




\section{Introduction}

Lenvatinib (LEN) is an oral multitarget tyrosine kinase inhibitor (TKI) directed toward the kinase activities of vascular endothelial growth factor (VEGF) receptors VEGFR1, 2, and 3. It also inhibits other proangiogenic and oncogenic pathways, including fibroblast growth factor receptors (FGFR1-4), the platelet derived growth factor receptor (PDGFRa), KIT proto-oncogenes, and RET [1]. It is currently used for advanced, radioiodine refractory differentiated thyroid cancer (RAI-R DTC), and it has been recently approved also for the treatment of patients with advanced or unresectable hepatocellular carcinoma (HCC) [2]. In RAI-R DTC patients on LEN treatment, several adverse events (AEs) have been reported in almost all treated patients, including hypertension, diarrhea, decreased appetite, decreased weight, fatigue, and proteinuria [3]. Most grade 3-4 adverse reactions occur during the first 6 months of treatment and require reduction of dosage and drug interruption or withdrawal. Among gastrointestinal events, diarrhea is the most frequently reported (range $45-67 \%$ any grade), but nausea, vomiting, and decreased appetite have been also described in several patients (range $18-75 \%$, any grade), in both registrative (SELECT) and real-life studies [1,3-6]. These events all contribute, together with dysgeusia, to the critical weight loss observed, particularly during the first 6 months of treatment in these patients [7]. The European Medicine Agency reports another gastrointestinal complication of LEN treatment, again possibly contributing to weight loss, which is cholecystitis, with or without stones (frequencies from $\geq 1 / 100$ to $<1 / 10$ ), but this $\mathrm{AE}$ is reported as extremely rare (0.4\%) in the SELECT trial [1], and it is even not mentioned in the following real-life studies [3-6]. Indeed, cholecystitis, with or without gallstones, has been only recently described during treatment with LEN in two studies dealing with RAI-R TC, with a prevalence of 14.7 and $8 \%[8,9]$. In the former study, LEN was always withdrawn and cholecystectomy performed in all patients [8], whereas no information on the management was reported in the latter study [9]. In recent years, cases of gallbladder disorders, mostly acalculous cholecystitis, have been described during treatment with some TKIs, such as imatinib [10], motesanib [11, 12], and sunitinib $[13,14]$. Clinical presentation may be similar in the cholecystitis with gallstones and in acalculous cholecystitis and includes right upper quadrant pain with tenderness to palpation, positive Murphy's sign and sometimes fever. Associated complaints may include nausea, vomiting, and anorexia; jaundice is unusually early in the course of acute cholecystitis (AC) but may occur when edematous inflammatory changes involve the bile ducts and surrounding lymph nodes [15]. Diagnosis of AC requires analysis of the clinical history and physical examination, laboratory tests and the association of abdominal imaging, such as computed tomography (CT) and/or ultrasound scan US showing gallbladder wall thickening or edema and/or pericholecystic fluid, and in some cases, the presence of gallstones. Acute acalculous cholecystitis can rise more concerns compared with cholecystitis with stones, since it can be complicated by gangrene, empyema, abscess formation, or perforation and is associated with high mortality rate, and early cholecystectomy is usually recommended [16]. However, like other surgical procedures, cholecystectomy can be extremely risky for these fragile patients, also because TKIs, including LEN, should be discontinued 5-7 days before surgery and restart after wound healing, 7-10 days after surgery.

Thus, considering its predicted strong clinical implications, we evaluated the prevalence and the clinical presentation of AC in our consecutive series of RAI-R DTC patients treated with LEN. Moreover, we verified whether a conservative approach could be successfully proposed, thus avoiding surgery and long-lasting treatment withdrawal.

\section{Patients and Methods}

We retrospectively analyzed the clinical records of our patients with RAI-R DTC who were on LEN treatment $(n=15)$ at the time of writing, April 2020. Two of them had cholecystectomy for gallstones before starting LEN treatment and were therefore excluded from the analysis. In the remaining 13 patients $(5 \mathrm{M}$ and $8 \mathrm{~F}$, median age at LEN start 62.2 years, range $21-84$ years), no radiological gallbladder alterations were found at the contrast-enhanced CT scan at baseline, immediately before the start of LEN. None of the patients were taking concomitant medications promoting cholecystitis (e.g., fibrates, HMG-CoA reductase inhibitors, thiazide diuretics, ceftriaxone, and anticoagulant treatment) or with interactions with LEN. Patient \#B was taking postmenopausal low-dosage estrogen therapy since 20 months. All patients were evaluated monthly during the first 4 months of treatment and thereafter at 2 -month intervals for up to 1 year. Biochemical evaluation included complete blood count, electrolytes, alanine aminotransferase $(\mathrm{ULN}=40 \mathrm{U} / \mathrm{L})$, aspartate aminotransferase $(\mathrm{ULN}=40 \mathrm{U} / \mathrm{L})$, total and conjugated bilirubin (ULN $=1.15$ and $0.2 \mathrm{mg} / \mathrm{dL}$, respectively), $\gamma \mathrm{GT}(\mathrm{ULN}=40 \mathrm{U} / \mathrm{L}$ female, $\mathrm{ULN}=60 \mathrm{U} / \mathrm{L}$ male), alkaline phosphatase $(\mathrm{ULN}=105 \mathrm{U} / \mathrm{L})$, calcium and albumin, lipase $(\mathrm{ULN}=70 \mathrm{U} / \mathrm{L})$, and amylase $(\mathrm{ULN}=160 \mathrm{U} / \mathrm{L})$. A contrast-enhanced CT was done at 3-6-month intervals during follow-up, in order to assess LEN treatment efficacy. In patients who developed hepatobiliary symptoms, further imaging was performed by contrast-enhanced CT and/or US, which were repeated during the follow-up for the evaluation of the radiological course. None of 


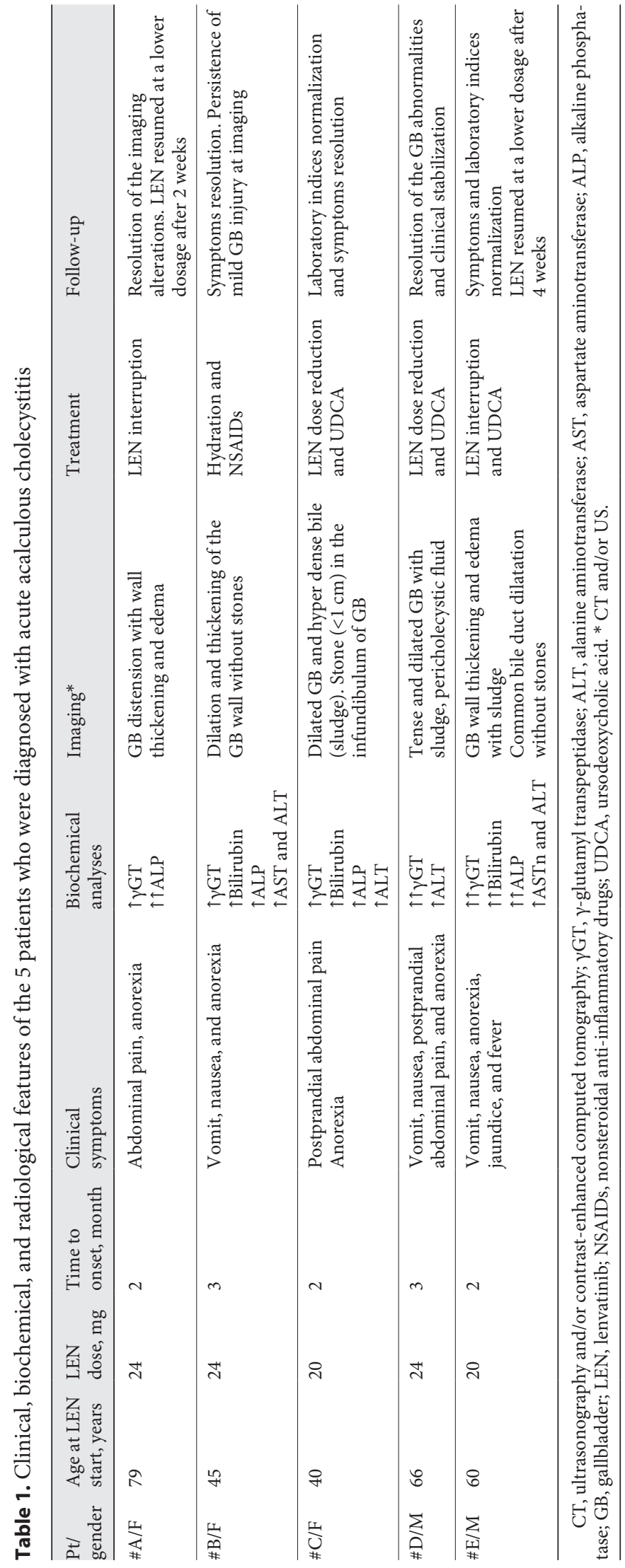

these patients had a diagnosis of liver metastases neither before starting LEN therapy nor during subsequent radiological followup, and there was no clinical evidence and/or history of gallbladder diseases. All procedures were performed in accordance with the ethical standards of the Research Committee of the Istituto Auxologico Italiano IRCCS and with the 1964 Helsinki Declaration and its later amendments.

Statistical analyses were done by means of $\chi^{2}$ and $t$ test, as appropriate. Statistical significance was defined as $p<0.05$. All statistical analyses were performed using SPSS version 8.0 for Windows and MedCalc Software version 11.6.1 for Windows.

\section{Results}

After a median time of 2 months (range 2-3 months) from the start of treatment, $5(3 \mathrm{~F}$ and $2 \mathrm{M})$ out of $13 \mathrm{pa}-$ tients (38.4\%, 95\% Confidence Interval 12-64.8\%) complained of gastrointestinal symptoms suggestive of cholecystitis, leading to further clinical and radiological investigations. The severity of this AE was scored according to the National Cancer Institute Common Terminology Criteria for Adverse Events, as mild/moderate (grade $1-2$ ) in 3 subjects and severe and/or medically significant (grade 3 ) in the remaining 2 patients. The clinical features, biochemical analyses, and radiological imaging of symptomatic cases are summarized in Table 1 . In particular, acute abdominal pain in the upper abdominal quadrant, right hypochondrium and/or epigastrium was reported by 3 patients, mostly as a post-prandial symptom or occurring during night' rest. In addition, nausea and vomiting were present in 3 subjects, while anorexia was reported by all patients. In 1 patient (\#E), there was a rapid worsening of general conditions, with great increase in cholestatic enzymes, fever, and jaundice requiring hospitalization.

The remaining 4 symptomatic patients all had an increase of $\gamma \mathrm{GT}$ levels (median value at 2 months $74.5 \mathrm{U} / \mathrm{L}$, range 50-116 U/L), including 1 with also alkaline phosphatase increase (\#A), 1 with also aminotransferases increase (\#D), and 2 with the association of alkaline phosphatase, conjugated bilirubin, and serum aminotransferases increase (\#B and \#C). No patient had alterations of either inflammation indices, such as leukocytosis, PCR or VES, or serum lipase and amylase, electrolytes, calcium, and albumin. Interestingly, $\gamma \mathrm{GT}$ increase was observed in all patients starting from the second month of LEN (Fig. 1). Since clinical and laboratory findings alone may be insufficient to make a differential diagnosis, we further performed imaging procedures. In all patients, CT and US were suggestive for AC, with the typical gallbladder 


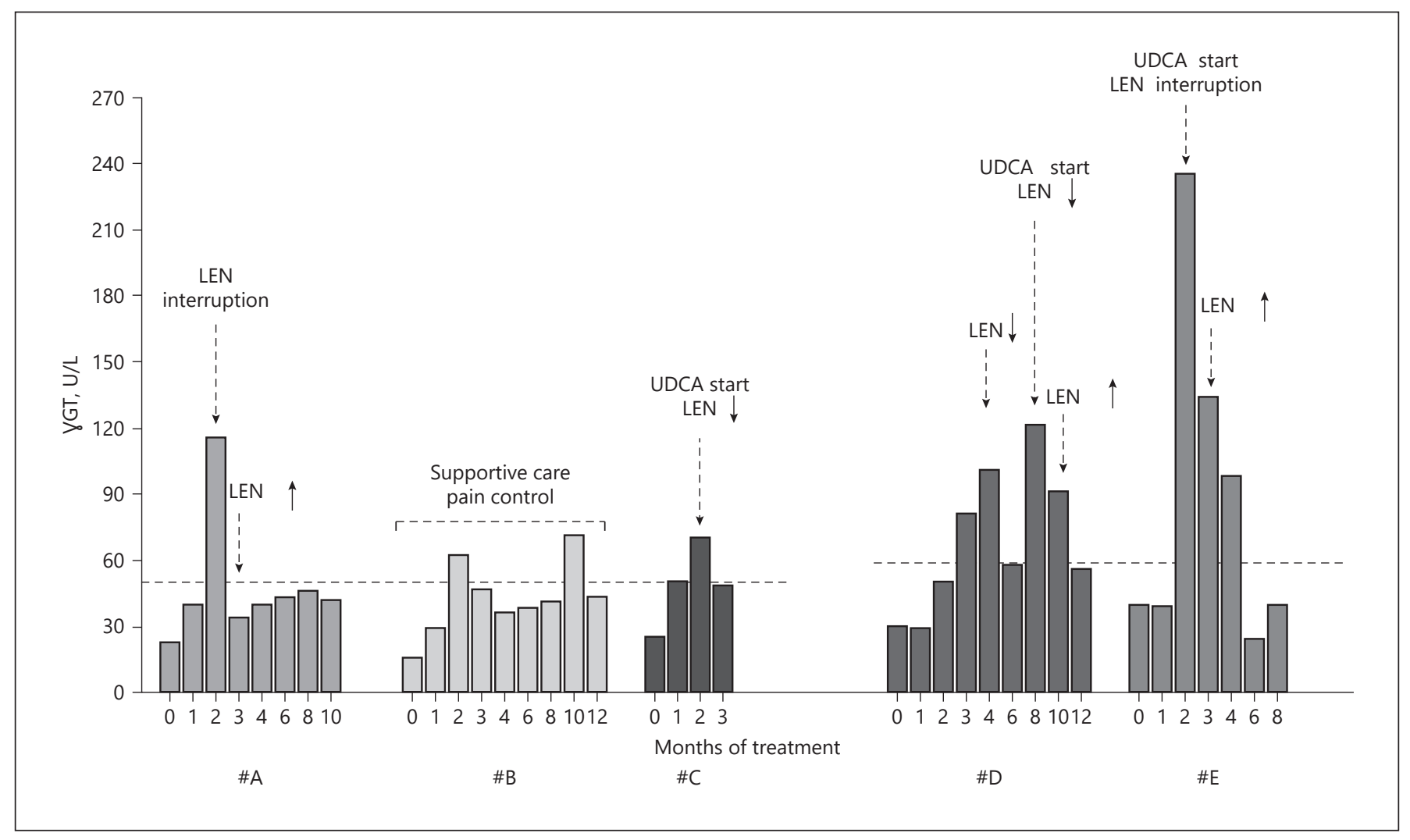

Fig. 1. Trend of $\gamma$ GT levels during LEN treatment in patients with RAI-R DTC. In all cases, $\gamma$ GT rose above the normal range after 2-3 months of treatment. The dotted lines indicate the upper limit of normal for females (\#A, $\# B$, and $\# \mathrm{C})(\mathrm{ULN}=40 \mathrm{U} / \mathrm{L})$ and males $(\# \mathrm{D}$ and $\# \mathrm{E})(\mathrm{ULN}=60 \mathrm{U} / \mathrm{L})$. The increase $(\uparrow)$ or decrease $(\downarrow)$ of LEN dosage is also reported. LEN, lenvatinib; RAI-R DTC, radioiodine refractory differentiated thyroid cancer; UDCA, ursodeoxycholic acid.

wall thickening and/or edema ( $>3 \mathrm{~mm})$, pericholecystic fluid, gallbladder distension $(>5 \mathrm{~cm})$, and, in $3 / 4$ cases, hyper dense bile (sludge). In 1 subject, a single small stone $(9 \mathrm{~mm})$ located in the infundibulum of gallbladder was associated with biliary sludge, while in the patient with the highest increase in cholestasis indices, fever, and jaundice $(\# \mathrm{E})$, a common bile duct dilatation $(16 \mathrm{~mm})$ was found in the absence of gallstones. In this subject, contrast-enhanced CT also showed a neo-enlargement of the pancreas head, which was submitted to a fine needle aspiration biopsy by an echo-endoscopic procedure, with no malignant findings at cytology.

The clinical characteristics and laboratory investigations of the 5 patients with AC were compared with the remaining 8 subjects without gallbladder disease treated with LEN (Table 2). With the limits related to the relatively low number of cases, no statistically significant differences were found neither in the age at the start of LEN treatment nor in the starting dose of LEN, nor in the body weight at baseline, nor in the body weight reduction after 2 months of treatment. There was a tendency toward a higher prevalence of symptoms such as abdominal pain, vomiting/nausea, and anorexia in the patients who developed AC, but the only statistically significant differences were observed for biliary enzymes levels ( $\gamma \mathrm{GT}$, alkaline phosphatase, and total bilirubin) and for alanine aminotransferase levels, which were higher in patients with AC with respect to asymptomatic cases.

In order to evaluate a possible relationship between weight loss, frequently observed during the first months of treatment, and gallbladder disorder, weight and $\gamma \mathrm{GT}$ trends were compared at baseline and at different times of follow-up (Fig. 2). Interestingly, 2 subjects (\#D and \#E) diagnosed with AC and biliary sludge showed a high reduction in body weight during the first months of therapy, with a delta reduction of 12.5 and $10 \mathrm{~kg}$, respectively. In both cases, the rapid weight loss was followed by the appearance of gastrointestinal symptoms, significant lab- 
Table 2. Clinical and biochemical features in patients with RAI-R DTC developing or not an acute acalculous cholecystitis during treatment with LEN

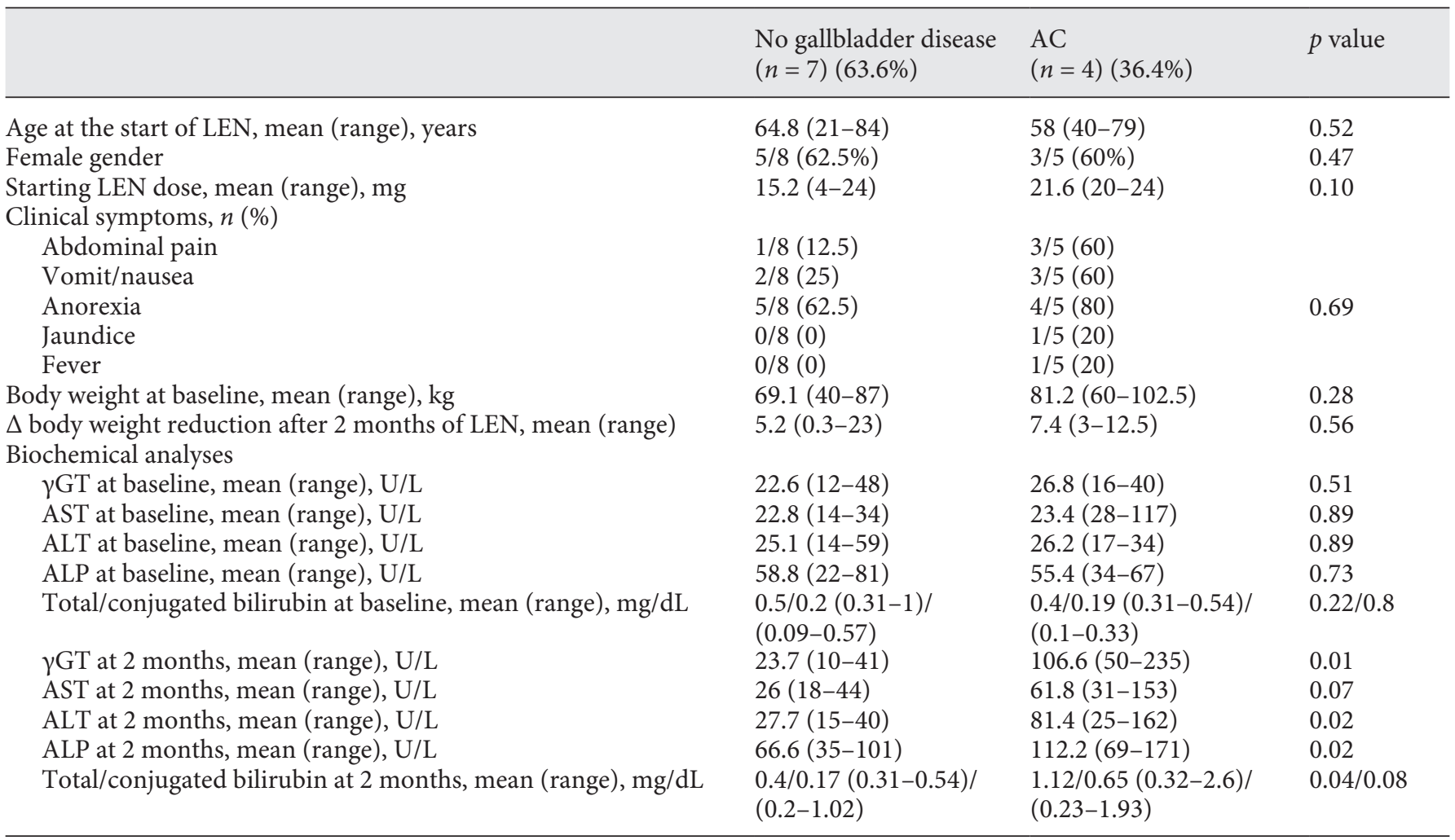

AC, acute cholecystitis; LEN, lenvatinib; RAI-R DTC, radioiodine refractory differentiated thyroid cancer; AST, aspartate aminotransferase; ALT, alanine aminotransferase; ALP, alkaline phosphatase.

oratory changes, and positive CT/US images consistent with AC. In the other 3 patients, the reduction in body weight was less pronounced at the time of the onset of symptoms and of radiological diagnosis (\#A: $\Delta$ weight reduction $-3.2 \mathrm{~kg}$ at 2 months; \#B $\Delta$ weight reduction: -3.5 $\mathrm{kg}$ at 2 months; \#C $\Delta$ weight reduction: $-3 \mathrm{~kg}$ at 2 months). These subjects were initially treated with LEN $24 \mathrm{mg}$ (\#A, $\# \mathrm{~B}$, and $\# \mathrm{D}$ ) and $20 \mathrm{mg}$ (\#C and \#E)/daily.

The management of these patients varied according to the degree of the clinical, biochemical, and radiologic features, though our main aim was always to avoid cholecystectomy. In 1 patient (\#B), we performed only a supportive care with hydration, fasting, and pain control with nonsteroidal anti-inflammatory drugs achieving a complete regression of clinical symptoms and improvement of the laboratory indices, though persistence of mild gallbladder injury was reported at the CT scan performed at 12 months (Fig. 3). In 2 patients with biliary sludge and cholelithiasis (\#D and $\# \mathrm{C}$, respectively), LEN dosage reduction was needed, in addition to medical support, to obtain clinical stabilization (Fig. 1). In particular, in the first subject (\#E), the dose was reduced from 24 to $20 \mathrm{mg} /$ day after 4 months of treatment and further reduced to 14 $\mathrm{mg} /$ day at month 8 due to a further increase of $\gamma \mathrm{GT}$ levels associated with nausea and vomiting; after 1 month of reduced LEN dose, ursodeoxycholic acid (UDCA) was started, and the final dosage of $20 \mathrm{mg} /$ day was progressively re-established and maintained (Fig. 3). In patient \#C, with gallbladder stone and massive biliary sludge, despite mild gastrointestinal symptoms, we reduced LEN from 20 to 20 and $14 \mathrm{mg}$ every other day and associated the litholytic therapy with UDCA. Finally, the last 2 cases (\#A and \#E), LEN interruption was needed for 2 and 4 weeks, respectively. Thereafter, the treatment was resumed at a lower dosage, and the control CT scan performed after 3 months showed resolution of the gallbladder abnormalities (Fig. 3). In patient \#E, who displayed extremely high $\gamma$ GT levels, UDCA treatment was associated and maintained during the whole follow-up. 

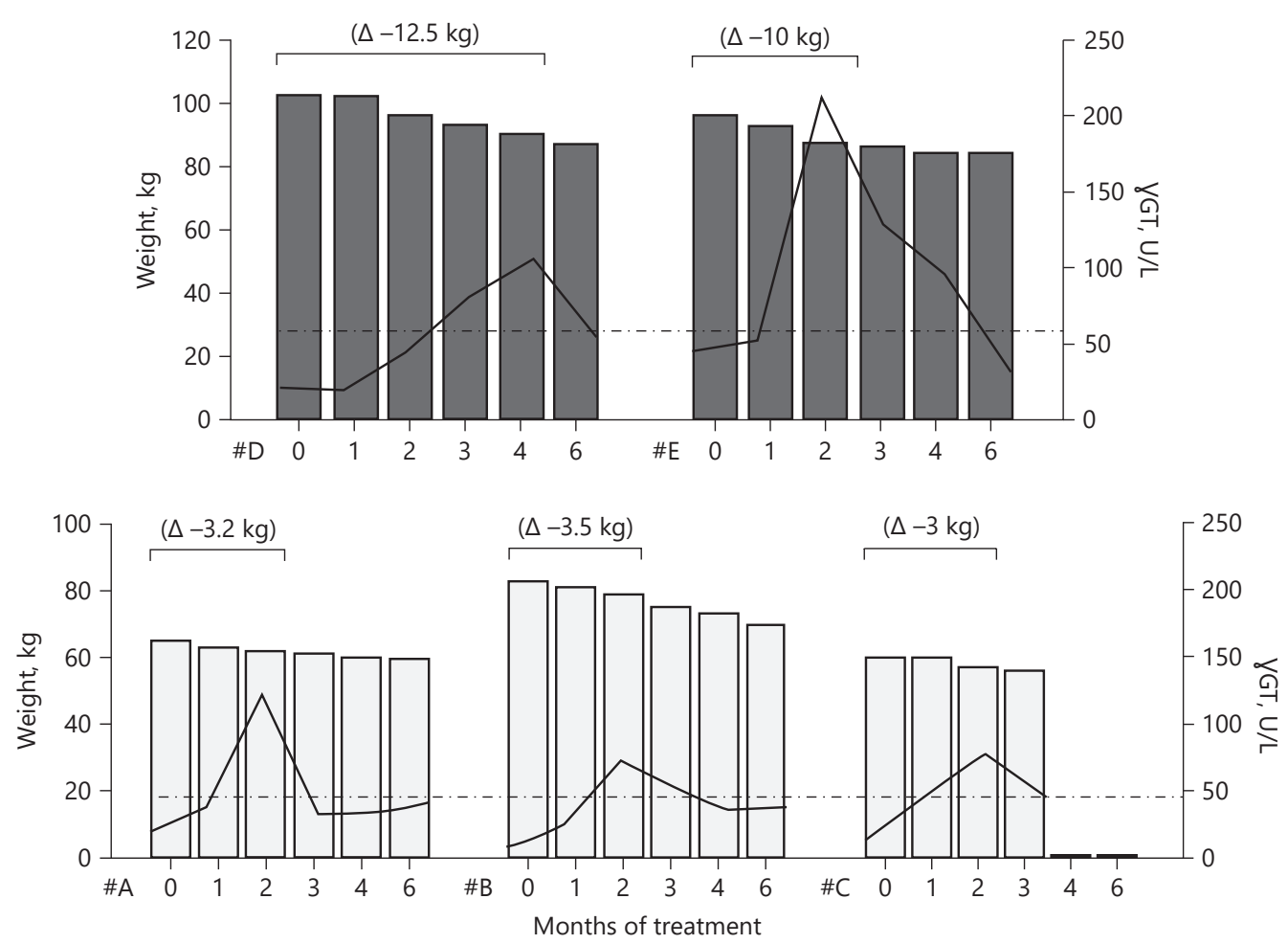

Fig. 2. Trend of $\gamma \mathrm{GT}$ levels and weight in the 5 patients with acute acalcolous cholecystitis. $\gamma$ GT levels increased above the normal range after 2-3 months of LEN, corresponding to the decrease in body weight. LEN, lenvatinib.

\section{Discussion}

Clinical, biochemical, and radiological features suggestive of AC were found in $38.4 \%$ (5/13) of our patients with RAI-R DTC on LEN treatment. In all cases, this AE occurred after 2-3 months of treatment, and the AC was lithiasic in only $1 / 5$ cases. The prevalence found in the present series is higher than that reported in the SELECT registrative trial $(0.4 \%)$ [1] and in the 2 recent studies $(14.7$ and $8 \%)[8,9]$. This could be certainly due to the limited number of patients included but could also indicate that this AE, though associated with a high morbidity, is frequently misdiagnosed, since the specific signs, such as the right upper quadrant pain, are not always present and the symptoms such as anorexia, nausea, and vomiting have a low specificity.

Differently from lithiasic $\mathrm{AC}$ resulting from the cystic duct obstruction by gallstones, acalculous AC is characterized by gallbladder stasis and ischemia, which then cause a local inflammatory response [15]. In particular, acalculous AC is a typical severe complication in hospitalized and critically ill patients with multiple risk factors like coronary heart disease, diabetes mellitus, immunosuppression, surgery, burns, sepsis, or AIDS [16], but also some antineoplastic target therapies can predispose to its development [13]. Several hypotheses have been formulated to explain the occurrence of acalculous AC during treatment with TKIs. First, the blockage exerted on the VEGF signaling pathway can ultimately result in an increased platelet activation and arterial thromboembolism via the reduction of prostaglandin I-2 and nitric oxide [17]. The arteriolar thrombosis in gallbladder could lead to ischemia and acalculous AC, as documented in a patient with HCC treated with sorafenib [18]. Furthermore, since cholangiocytes express VEGFR-2 and VEGFR-3 and secrete VEGF which has an important role in inducing their proliferation in response to cholestasis [19], drugs inhibiting VEGF-R are predicted to cause biliary diseases. The antiangiogenic activity of TKIs at the epithelium and muscularis layers of the gallbladder may also contribute to necroinflammatory alterations observed in cholecystitis [14], especially for those compounds, like LEN, which have a predominantly biliary excretion re- 


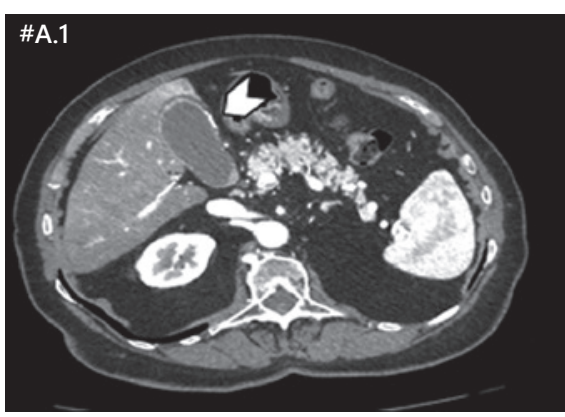

$2^{\circ}$ month of treatment ( $24 \mathrm{mg} /$ day)
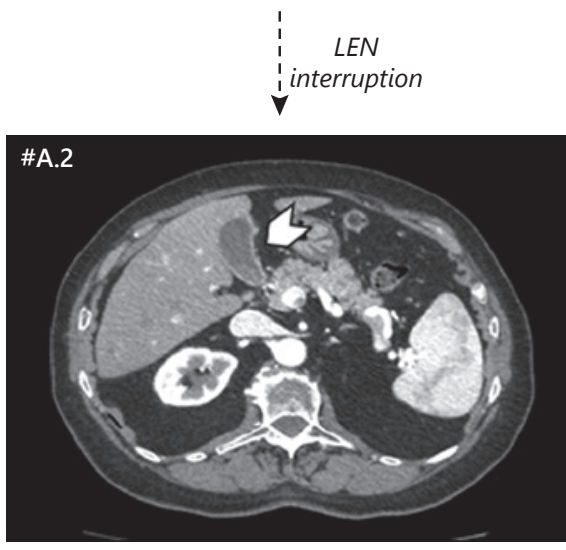

$3^{\circ}$ month of treatment (14 mg/day)

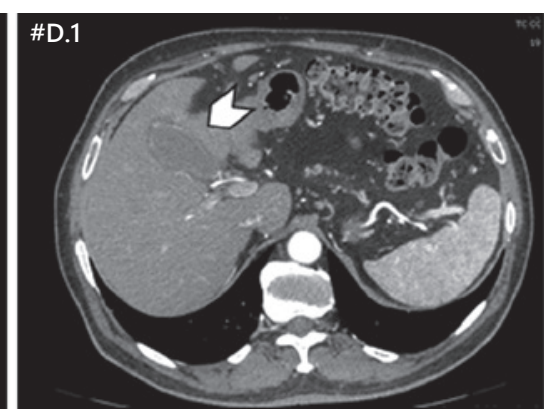

$3^{\circ}$ month of treatment ( $24 \mathrm{mg} /$ day)

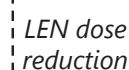

reduction

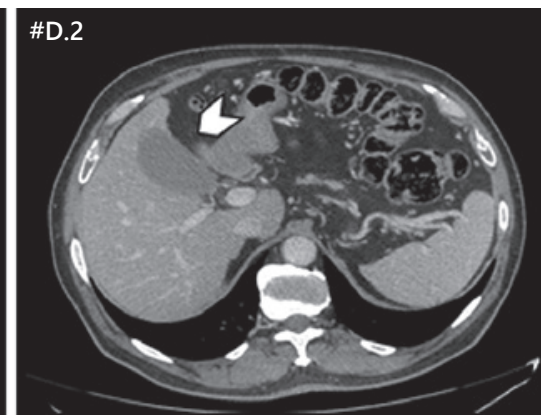

$10^{\circ}$ month of treatment ( $4 \mathrm{mg} /$ day)

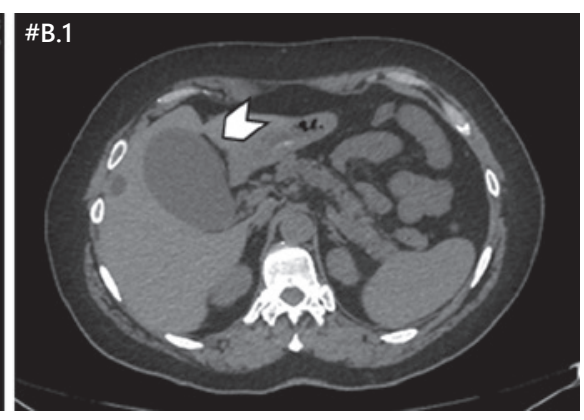

$3^{\circ}$ month of treatment ( $24 \mathrm{mg} /$ day)

Symptomatic treatment

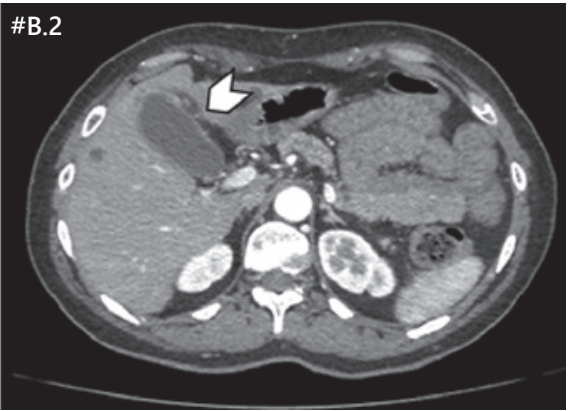

$12^{\circ}$ month of treatment ( $24 \mathrm{mg} /$ day)
Fig. 3. Evolution of the acute acalcolous cholecystitis upon different therapeutic interventions in 3 patients. \#A Panel 1: edema and thickening of the gallbladder wall with exudate. Panel 2: restitutio ad integrum after 2 weeks of LEN interruption. \#D Panel 1: edema and thickening of the gallbladder wall. Panel 2: major improve- ment after LEN dose reduction. \#B Panel 1: large volume increase of the gallbladder. Panel 2: gallbladder injury persists, in the absence of clinical and/or biochemical alterations, after treatment with hydration and nonsteroidal anti-inflammatory drugs. LEN, lenvatinib. sulting in the accumulation of the drug and its metabolites in bile fluids. The above mentioned hypothesized mechanisms could have a prevalent role in acalculous AC, whereas the possibility that TKI drugs may play a lithogenic effect is less defined. Nevertheless, it is worth to mention that gallbladder diseases can certainly contribute to the weight loss experienced by $50-90 \%$ of patients treated with LEN for RAI-R DTC, particularly during the first 6 months of treatment (BMI delta reduction $9.7 \%$, range 4-24.7\%) [7], but it could also be the consequence of the weight loss in a sort of vicious cycle. Indeed, in our series, the highest levels of $\gamma \mathrm{GT}$, a specific marker of gallbladder disease, corresponded to the highest weight reduction. Consistently, it has been reported that $10-20 \%$ of subjects undergoing a rapid weight reduction $(>1.5 \mathrm{~kg} /$ week) develop gallstones. Even biliary sludge is often found incidentally in situations leading to increased gall- bladder stasis and/or concurrent change of biliary composition, such as those associated with rapid weight loss (e.g., very-low-calorie diet and bariatric surgery) [20]. Recent European recommendations suggest to start a temporary treatment with the anti-lithiasic UDCA in these conditions [20]. Accordingly, the treatment with UDCA was extremely effective in 3 of our patients, presenting with biliary sludge associated in 1 case with a small stone.

The development of AC seems to be strictly correlated to LEN treatment since our patients did not show any gallbladder alteration at baseline. On the other hand, it seems that no correlation exists between the age at the start of LEN treatment and the development of symptomatic AC. Similarly, the starting dose of LEN did not correlate neither with the onset nor with the severity of the gallbladder injury, though dosage reduction was always associated with a clinical, biochemical, and radiological 
improvement. In all AC patients, we observed the early increase of biliary enzymes and in particular of $\gamma \mathrm{GT}$ levels, indicating that the latter is the most important and most accurate marker to assess the functional status of the gallbladder in patients treated with LEN, and that it should be routinely measured in order to allow a prompt diagnosis and a more effective treatment. Indeed, the increase in biliary enzymes was the only clinical and biochemical parameter which was statistically different between $\mathrm{AC}$ cases and asymptomatic patients.

Among our cases, only 1 presented fever in association with gastroenterological symptoms, and leukocytosis was never observed at the time of diagnosis. These findings are not surprising since both fever and leukocytosis are frequently absent during AC [21]. Moreover, it should be also noted that the decrease in lymphocyte count is included among AE of LEN treatment, indicating that this biochemical parameter cannot be used as a diagnostic indicator in these particular patients.

Early management is mandatory because, if untreated, acalculous AC can be complicated with high mortality due to gangrene and the perforation of the gallbladder [22]. Consistently, in a recent report, the 5 patients who developed cholecystitis during LEN were all treated with cholecystectomy [8]. In the present series, cholecystectomy has been avoided in all cases, and a tailored treatment was adopted, based on the severity of symptoms, the degree of alteration of biochemical parameters, and the imaging information. In particular, in the presence of $\mathrm{mild} /$ moderate symptoms and/or laboratory abnormalities (grade 1-2) we decided to carry out only a supportive medical treatment, whereas 2 patients showing more evident radiological alterations and severe symptoms were treated by short-lasting reduction of LEN dosage associated with UDCA, with an acceptable clinic control. In 2 patients, LEN treatment was stopped for 2-4 weeks due to severe symptoms (grade $>3$ ) compromising adequate nutrition and associated with major radiological findings.

In conclusion, our data show the frequent occurrence of AC, mainly acalculous, during treatment with LEN in patients with RAI-R DTC. The prevalence found in this limited series is higher than those previously reported, indicating that additional evidences in larger series are certainly needed, particularly to confirm or to establish the actual impact of this AE. Nevertheless, we believe that some important indications can be derived from our data. First, we observed that this AE usually associates with weight loss during the first months of treatment, and contributes to further decrease in body weight. Thus, it is mandatory to limit weight loss by introducing dietary and rehabilitative supports. The biliary enzymes, or at least $\gamma \mathrm{GT}$, should be routinely prescribed before the start and during the first 3 months of treatment, and immediately when a pronounced weight loss occurs during follow-up, in order to obtain a prompt diagnosis. US monitoring should be added to the follow-up in case of $\gamma$ GT elevation. Second, our data suggest that treatment with UDCA is highly beneficial in the clinical and radiological resolution of the gallbladder injury, and, if associated with an accurate titration of LEN dosage, can limit or avoid the need for drug withdrawal and cholecystectomy.

\section{Statement of Ethics}

The paper is exempt from ethical committee approval since all procedures done and reported in the present study are included in the common clinical practice. All the subjects included gave their written informed consent for the use of their clinical data.

\section{Conflict of Interest Statement}

M.D.S., C.C., S.D.L., M.P., M.V., and L.P. have no conflicts of interest to declare. L.F. has a consultant relationship with Eisai.

\section{Funding Sources}

The authors did not receive any funding.

\section{Author Contributions}

Marta Di Stefano, Carla Colombo, and Simone De Leo: design of the study, data collection and analyses, and writing of manuscript draft. Carla Colombo, Marta Di Stefano, and Michela Perrino: data collection and analyses. Mauro Viganò: hepatological data and revision of the manuscript. Luca Persani: data interpretation and revision of the final manuscript. Laura Fugazzola: design of the study, data interpretation, and writing of the final manuscript.

\section{References}

1 Schlumberger M, Tahara M, Wirth LJ, Robinson B, Brose MS, Elisei R, et al. Lenvatinib versus placebo in radioiodine-refractory thyroid cancer. N Engl J Med. 2015;372(7):621-30.

2 Kudo M, Finn RS, Qin S, Han KH, Ikeda K, Piscaglia F, et al. Lenvatinib versus sorafenib in first-line treatment of patients with unresectable hepatocellular carcinoma: a randomised phase 3 non-inferiority trial. Lancet. 2018 Mar 24;391(10126):1163-73.

3 Fugazzola L, Elisei R, Fuhrer D, Jarzab B, Leboulleux S, Newbold K, et al. 2019 European thyroid association guidelines for the treatment and follow-up of advanced radioiodinerefractory thyroid cancer. Eur Thyroid J. 2019 Oct;8(5):227-45. 
4 Nervo A, Gallo M, Samà MT, Felicetti F, Alfano M, Migliore E, et al. Lenvatinib in advanced radioiodine-refractory thyroid cancer: a snapshot of real-life clinical practice. Anticancer Res. 2018 Mar;38(3):1643-49.

5 Berdelou A, Borget I, Godbert Y, Nguyen T, Garcia ME, Chougnet CN, et al. Lenvatinib for the treatment of radio-iodine refractory thyroid cancer in real-life practice. Thyroid. 2017 Nov 27.

6 Molina-Vega M, García-Alemán J, SebastiánOchoa A, Mancha-Doblas I, Trigo-Pérez JM, Tinahones-Madueño F. Tyrosine kinase inhibitors in iodine-refractory differentiated thyroid cancer: experience in clinical practice. Endocrine. 2018 Feb;59(2):395-401.

7 De Leo S, Colombo C, Di Stefano M, Dubini A, Cozzi S, Persani L, et al. Body composition and leptin/ghrelin levels during lenvatinib for thyroid cancer. Eur Thyroid J. 2020 Jan;9(1): $1-10$.

8 Nervo A, Ragni A, Gallo M, Ferraris A, Fonio P, Piovesan A, et al. Symptomatic biliary disorders during lenvatinib treatment for thyroid cancer: an underestimated problem. Thyroid. 2020 Feb;30(2):229-36.

9 Aydemirli MD, Kapiteijn E, Ferrier KRM, Ottevanger PB, Links TP, van der Horst-Schrivers ANA, et al. Effectiveness and toxicity of lenvatinib in refractory thyroid cancer: Dutch real-life data. Eur J Endocrinol. 2020 Feb; 182(2):131-8
10 Yeh CN, Chen TW, Liu FY, Jan YY, Chen MF. Genetic changes in advanced gastrointestinal stromal tumor (GIST) patients during imatinib mesylate treatment. Langenbecks Arch Surg. 2006;391(6):615-21.

11 Schlumberger MJ, Elisei R, Bastholt L, Wirth LJ, Martins RG, Locati LD, et al. Phase II study of safety and efficacy of motesanib in patients with progressive or symptomatic, advanced or metastatic medullary thyroid cancer. J Clin Oncol. 2009;27(23):3794-801.

12 Sherman SI, Wirth LJ, Droz JP, Hofmann M, Bastholt L, Martins RG, et al., Motesanib diphosphate in progressive differentiated thyroid cancer. N Engl J Med. 2008;359(1):3142.

13 Tirumani SH, Krajewski KM, Shinagare AB, Jagannathan JP, Ramaiya NH. Gallbladder complications associated with molecular targeted therapies: clinical and imaging features. Clin Imaging.2014;38(1):50-5.

14 Gomez-Abuin G, Karam AA, Mezzadri NA, Bas CA. Acalculous cholecystitis in a patient with metastatic renal cell carcinoma treated with sunitinib. Clin Genitourin Cancer. 2009; $7(1): 62-3$.

15 Jameson JL, Fauci AS, Kasper DL, Hauser SL, Longo DL, Loscalzo J. Harrison's principles of internal medicine. 12th ed. New York: McGraw Hill; 2018. Vol. 1 and 2.
16 Huffman JL, Schenker S. Acute acalculous cholecystitis: a review. Clin Gastroenterol Hepatol. 2010 Jan;8(1):15-22.

17 Elice F, Rodeghiero F, Falanga A, Rickles FR. Thrombosis associated with angiogenesis inhibitors. Best Pract Res Clin Haematol. 2009; 22(1):115-28.

18 Sanda M, Tamai H, Deguchi H, Mori Y, Moribata K, Shingaki N, et al. Acalculous cholecystitis in a patient with hepatocellular carcinoma on sorafenib. ISRN Gastroenterol. 2011;2011:201529.

19 Gaudio E, Barbaro B, Alvaro D, Glaser S, Francis H, Ueno Y, et al., Vascular endothelial growth factor stimulates rat cholangiocyte proliferation via an autocrine mechanism. Gastroenterology. 2006;130(4):1270-82.

20 European Association for the Study of the Liver (EASL). EASL Clinical Practice Guidelines on the prevention, diagnosis and treatment of gallstones. J Hepatol. 2016;65(1): 146-81.

21 Singer AJ, McCracken G, Henry MC, Thode HC, Cabahug CJ. Correlation among clinical, laboratory, and hepatobiliary scanning findings in patients with suspected acute cholecystitis. Ann Emerg Med. 1996;28(3):267-72.

22 Wang AJ, Wang TE, Lin CC, Lin SC, Shih SC. Clinical predictors of severe gallbladder complications in acute acalculous cholecystitis. World J Gastroenterol. 2003;9(12):2821-3. 